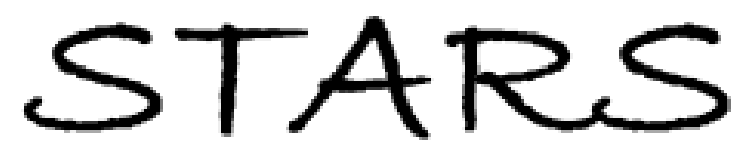

University of Central Florida

STARS

$1-1-2008$

\title{
Relaxation time effects on dynamic conductivity of alloyed metallic thin films in the infrared band
}

\section{J. Shelton}

University of Central Florida

T. Sun

University of Central Florida

J. C. Ginn

University of Central Florida

K. R. Coffey

University of Central Florida

G. D. Boreman

University of Central Florida

Find similar works at: https://stars.library.ucf.edu/facultybib2000

University of Central Florida Libraries http://library.ucf.edu

This Article is brought to you for free and open access by the Faculty Bibliography at STARS. It has been accepted for inclusion in Faculty Bibliography 2000s by an authorized administrator of STARS. For more information, please contactSTARS@ucf.edu.

\section{Recommended Citation}

Shelton, D. J.; Sun, T.; Ginn, J. C.; Coffey, K. R.; and Boreman, G. D., "Relaxation time effects on dynamic conductivity of alloyed metallic thin films in the infrared band" (2008). Faculty Bibliography 2000s. 978. https://stars.library.ucf.edu/facultybib2000/978

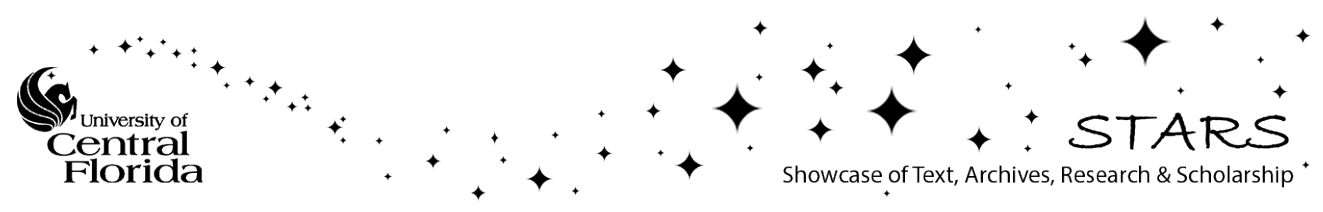




\section{Relaxation time effects on dynamic conductivity of alloyed metallic thin films in the infrared band}

Cite as: J. Appl. Phys. 104, 103514 (2008); https://doi.org/10.1063/1.3026717

Submitted: 07 August 2008. Accepted: 07 October 2008. Published Online: 19 November 2008

D. J. Shelton, T. Sun, J. C. Ginn, K. R. Coffey, and G. D. Boreman

\section{ARTICLES YOU MAY BE INTERESTED IN}

Altering infrared metamaterial performance through metal resonance damping Journal of Applied Physics 105, 074304 (2009); https://doi.org/10.1063/1.3093698

Terahertz conductivity of thin metal films

Applied Physics Letters 93, 051105 (2008); https://doi.org/10.1063/1.2968308

Electron mean free path in elemental metals

Journal of Applied Physics 119, 085101 (2016); https://doi.org/10.1063/1.4942216

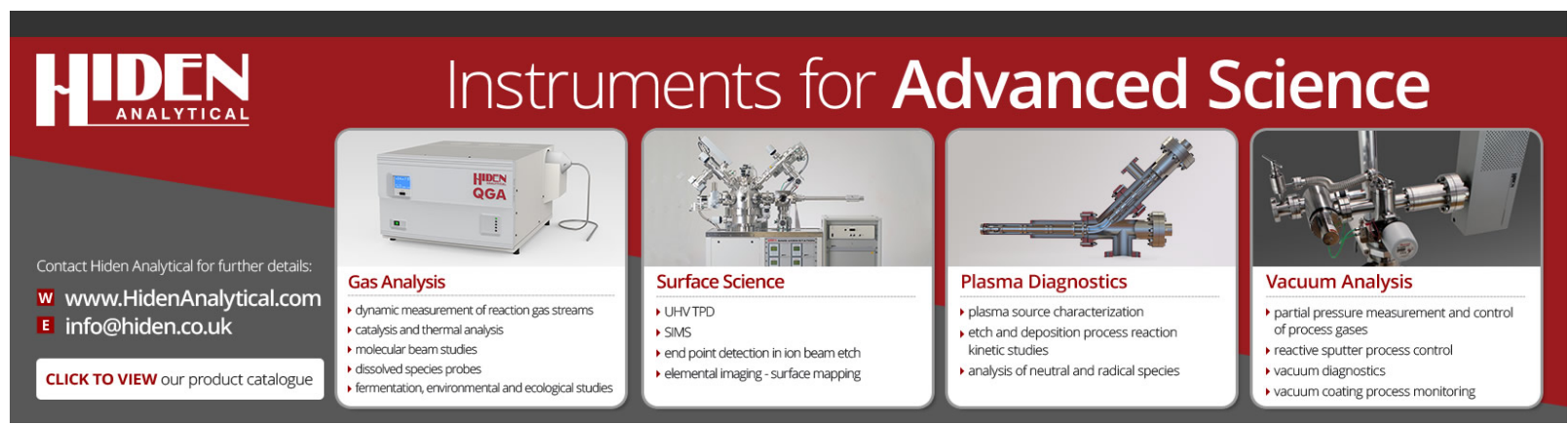




\title{
Relaxation time effects on dynamic conductivity of alloyed metallic thin films in the infrared band
}

\author{
D. J. Shelton, ${ }^{1, a)}$ T. Sun, ${ }^{2}$ J. C. Ginn, ${ }^{1}$ K. R. Coffey, ${ }^{2}$ and G. D. Boreman ${ }^{1}$ \\ ${ }^{1}$ CREOL, University of Central Florida, Orlando, Florida 32816, USA \\ ${ }^{2}$ AMPAC, University of Central Florida, Orlando, Florida 32816, USA
}

(Received 7 August 2008; accepted 7 October 2008; published online 19 November 2008)

\begin{abstract}
The behavior of nanoscale infrared antenna elements depends upon the dynamic conductivity of thin metallic films. Spectroscopic ellipsometer measurements of noble metal films show that when the product of the incident radiation frequency and the relaxation time is greater than unity, anomalous dynamic electron transport effects occur. In this regime electron scattering increases the conductivity of alloyed metallic films as demonstrated by ellipsometry measurements of films from the $\mathrm{Au}-\mathrm{Cu}$ system. A binary alloy thin film was fabricated with equal parts of $\mathrm{Au}$ and $\mathrm{Cu}$, and the dynamic conductivity was measured to be $300 \%$ larger than the high frequency conductivity of pure $\mathrm{Au}$ or pure $\mathrm{Cu}$ films at wavelengths in the 3-5 $\mu \mathrm{m}$ band. When electronic scattering is reduced, ellipsometer measurements of $\mathrm{Au}$ and $\mathrm{Cu}$ films taken near $4 \mathrm{~K}$ demonstrate that the IR conductivity decreases to $20 \%$ of the value measured at $300 \mathrm{~K}$ at wavelengths in the $3-5 \mu \mathrm{m}$ band. Using measured dc relaxation times, a model to explain deviations from Drude behavior was developed using the theory of the anomalous skin effect and frequency dependent relaxation time. This model was in quantitative agreement with the measured data. The ability to design an alloyed metallic thin film using a calculated ideal dc relaxation time to produce the greatest possible dynamic conductivity for infrared antennas and metamaterials was demonstrated. (c) 2008 American Institute of Physics. [DOI: 10.1063/1.3026717]
\end{abstract}

\section{INTRODUCTION}

The dynamic conductivity describes the propagation of a current wave on a metallic structure in response to incident electromagnetic radiation. As derived from the Drude model, dynamic conductivity is a complex number with the real part corresponding to Ohmic loss and the imaginary term corresponding to the permittivity of the metal. The permittivity is an important material property for near field surface plasmon resonance, but it has less impact on the behavior of antenna systems. Within the semiclassical framework considered in this paper, and limiting the discussion to polycrystalline metals, dynamic conductivity is a scalar quantity given in Eq. (1) below where $j$ is the current density, $E$ is electric field, and $\sigma$ is dynamic conductivity.

$$
j(\omega)=\sigma(\omega) \times E(\omega) .
$$

The 30-200 THz band is of particular interest to the IR sensing and metamaterials communities. These devices typically use noble metal and $\mathrm{Al}$ films with thicknesses on the order of $100 \mathrm{~nm}$, and the real part of dynamic conductivity is the main material property used in design and simulation. ${ }^{1-3}$ As predicted by the Drude model, the real part of conductivity decreases as the plasma frequency is approached from the low frequencies. This leads to an order of magnitude decrease in the dynamic conductivity of all metallic elements compared to dc values. Several experiments have also determined that there is no superconducting state at these frequencies, ${ }^{4,5}$ so limited electrical conductivity is an inescapable problem for IR antenna systems. At visible radiation

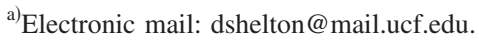

frequencies recent advances in surface plasmon polariton applications have made use of confined near field interactions to improve upon traditional antenna designs, which depend on dynamic conductivity. ${ }^{6}$ However the $30-200 \mathrm{THz}$ band is too far below the plasma frequency of the metals for this technology to translate to the IR. ${ }^{7,8}$ Therefore dynamic conduction in the IR band is a middle ground between the intrinsically high conductivity of the rf band and the ability to utilize resonant surface plasmons in the visible. This is the motivation to use other techniques to optimize dynamic conductivity in the IR to provide for higher performance antenna elements. In particular the signal to noise ratios of IR sensing antennas increase, and the resonant bandwidth of IR metamaterials decrease, with increasing dynamic conductivity.

Previous research has found that variation in the surface morphology of metals that are sufficiently thin to experience classical size effects has altered the dynamic conductivity in the IR for $\mathrm{Fe}^{9}$ as well as $\mathrm{Cu}$ films. ${ }^{10}$ However, reduction in surface roughness is not a feasible means to significantly increase dynamic conductivity for the films used in IR devices because the rms surface roughness in metal films deposited on prime polished silicon wafers is already low, (only $5 \AA$ as measured by atomic force microscopy).

Instead of limiting the investigation to the surface of the film the influence of the electronic mean free path within the film on dynamic conductivity may also be considered. According to the Drude model, the dynamic conductivity should depend upon the relaxation time. The relaxation time is defined as the ratio of the electronic mean free path $\left(\lambda_{\mathrm{mfp}}\right)$ to the Fermi velocity. Modifying the electronic mean free 
path by adding impurities or decreasing the temperature changes the relaxation time of the film and alters the dynamic conductivity within the radiation penetration depth (skin depth) while the surface scattering contribution may be unchanged. For free electrons in noble metals the relaxation time may be approximated within the skin depth as being isotropic. In the IR the skin depth encompasses a depth of around $10 \mathrm{~nm}$. The Drude model may be used to calculate the effect of changing relaxation time on dynamic conductivity in $\mathrm{Au}$ and $\mathrm{Cu}$ films deposited by physical vapor deposition (PVD) tools.

\section{THEORY}

\section{A. Drude model}

When electromagnetic radiation is incident on a metallic film, the dynamic conductivity may be expressed as in Eq. (2) where $\sigma_{0}$ is the dc conductivity and $\tau$ is the relaxation time. $^{11}$

$$
\sigma(\omega)=\frac{\sigma_{0}}{1-i \omega \tau}
$$

The real part of Eq. (2) is related to Ohmic loss and can be equated with the optical constants $n$ and $k$, which are the real and imaginary portions of the refractive index. ${ }^{11}$ This is shown below in Eq. (3).

$$
\sigma^{\prime}(\omega)=\frac{\sigma_{0}}{1+(\omega \tau)^{2}}=2 n k \varepsilon_{0} \omega
$$

Equation (3) will be of particular use for the interpretation of measured results of dynamic conductivity. The real part of the dynamic conductivity may be further rewritten to expand the dc conductivity in terms of relaxation time. This is shown in Eq. (4) where $n$ is the density of valence electrons, $e$ is the charge on the electron, and $m^{*}$ is the effective mass. Equation (4) assumes that the dc relaxation time is equivalent to the dynamic relaxation time. We will investigate the validity of this assumption.

$$
\sigma^{\prime}(\omega)=\frac{n e^{2} \tau}{m^{*}\left[1+(\omega \tau)^{2}\right]} .
$$

It is evident from Eq. (4) that in the case that the $\omega \tau$ product is much greater than unity the dynamic conductivity will be inversely proportional to the relaxation time. In this case dynamic conductivity decreases as the mean free path increases. Conversely, if the mean free path is decreased by impurity scattering, then the dynamic conductivity should increase. The maximum value for dynamic conductivity in Eq. (4) occurs when the $\omega \tau$ product is equal to unity. In the rf band a large $\tau$ is required to satisfy this condition, but in the IR band pure metal films have $\omega \tau$ products that are much greater than unity, so $\tau$ must be decreased to maximize the dynamic conductivity in the IR band.

The discussion in this paper has been limited to noble metals so as to avoid effects inconsistent with the Drude model that occur in transition metals for which the electronic mean free path is less than or equal to the Fermi wavelength.
In this limit transport must be examined using quantum mechanical models, and relaxation time effects may no longer be dominant. ${ }^{12}$

\section{B. Anomalous skin effect}

In addition to the Drude model the dynamic conductivity may be further understood in terms of the anomalous skin effect. As stated previously only electrons within the skin depth of the metal participate in dynamic conductivity. A further stipulation is that only the electrons that travel a mean free path within the skin layer may be considered carriers in dynamic conductivity. When skin depth is less than or nearly equal to the mean free path electrons with trajectories oriented normal to the surface will then no longer participate in dynamic conductivity. ${ }^{13}$ Such electrons were termed ineffective by Pippard in Ref. 14 and used to explain the anomalous skin effect at radio wave frequencies in noble metals at cryogenic temperatures. The fraction of effective electrons may then be derived from the Boltzmann transport equation $^{15}$ and the result is given as

$$
n_{\mathrm{eff}}=n \beta \frac{\delta^{\prime}}{\lambda_{\mathrm{mfp}}}=n \frac{1}{\tau} \frac{\beta^{2 / 3}}{V_{F}^{2 / 3}} \sqrt{\frac{2 m^{*}}{\omega n e^{2} \mu_{0}}},
$$

where $\delta^{\prime}$ is the anomalous skin depth and the ratio of $\delta^{\prime}$ to mean free path is given on the right side of the equation written in terms of constants from Eqs. (1)-(4). The unitless constant $\beta$ is related to surface roughness and is proportional to the mean free path times the ratio of the sharpness of surface features to the depth of surface features. ${ }^{16}$ If the mean free path is much larger than the skin depth then changes in skin layer scattering will not affect the dynamic conductivity. In this limit the dynamic conductivity is dependent on just the Fermi surface and the surface scattering. However, in the metals used for IR antennas this would not be significant because the mean free path is reduced due to defects and grain boundaries. Even at cryogenic temperatures the mean free path does not become very large compared to skin depth because of the high residual defect scattering present in thin films. In the regime where the mean free path is not much larger than the skin depth, scattering within the skin layer should increase dynamic conductivity by restoring the normal skin effect and increasing the effective carrier concentration toward its standard value of one free electron per atom. Similarly to the Drude model, the effective electron concentration in Eq. (5) is inversely proportional to relaxation time.

\section{Dynamic relaxation time}

In the Drude model the relaxation time is treated as a constant value that may be equated with the dc relaxation time. However, it has been observed experimentally in Ref. 17 that the relaxation time tends to vary as a function of frequency according to the form 


$$
\tau(\omega)=\frac{1}{a+b \omega^{2}}
$$

Clearly the constant term $a$ in Eq. (6) corresponds to the inverse of the dc relaxation time. Several explanations have been given for the quadratic term. In Ref. 18 dynamic conductivity is described by a two-carrier model in which the constant term is the dc relaxation time of carriers inside the crystalline grains, and the quadratic term is determined by a second distinct carrier type on the grain boundaries. A second explanation in Ref. 19 is that the quadratic term results from electron-electron scattering events that occur in all metals at high frequency and are independent of temperature and crystal defects. To completely describe the behavior of dynamic conductivity in the IR, a frequency-dependent relaxation time should be included in addition to the effective carrier concentration from the anomalous skin effect. Thus the ratio between measured dynamic conductivity and the Drude model $\gamma(\omega)$ in Eq. (7) may be formed by substituting Eq. (6) into Eq. (5). The validity of this equation may then be proven by comparison to data from dynamic conductivity experiments conducted at different temperatures and degrees of impurity scattering.

$$
\frac{\sigma(\omega)_{\text {measured }}}{\sigma(\omega)_{\text {Drude }}}=\gamma(\omega)=\left(\frac{1}{\tau_{\mathrm{dc}}}+b \omega^{2}\right) \frac{\beta^{2 / 3}}{V_{F}^{2 / 3}} \sqrt{\frac{2 m^{*}}{\omega n e^{2} \mu_{0}}} .
$$

\section{EXPERIMENTAL DETAILS}

Dynamic conductivity may be determined from optical constants measured by ellipsometry as shown in Eq. (3). Ellipsometric measurements are taken by reflecting circularly polarized IR radiation at an oblique angle from a thin film sample, and then measuring the polarization state of the reflected elliptically polarized radiation. In these experiments a J. A. Woollam Co. IR-VASE (variable angle spectroscopic ellipsometer) was used under standard ex situ conditions. In addition, the low temperature measurements were taken using an UHV cryostat attachment. Ellipsometry has been found to be a reliable method for measuring the optical properties of metal films in Refs. 20 and 21. When used in antenna simulations, optical constants and corresponding dynamic conductivities from Eq. (3) which were obtained via ellipsometry, have been found to improve the agreement between simulated and measured results for IR frequency selective surfaces. ${ }^{3}$ Therefore improvements in dynamic conductivity measured by ellipsometry can be assumed to induce an improvement in the performance of IR antenna systems. When multiple transparent layers are present in the sample a model is developed to fit the optical properties and thickness of the layers. For metallic films several skin depths thick, only the metal and any surface oxides are measured, so the model is straightforward. The films discussed in this paper, $\mathrm{Au}$ and $\mathrm{Cu}$, did not have any appreciable surface oxide layers. Noise caused by depolarized reflected radiation was low at only $1 \%-5 \%$ across the spectrum. Measurements were taken from 2 to $20 \mu \mathrm{m}$ and at angles of incidence ranging from $65^{\circ}$ to $75^{\circ}$. There were no significant changes in dy-

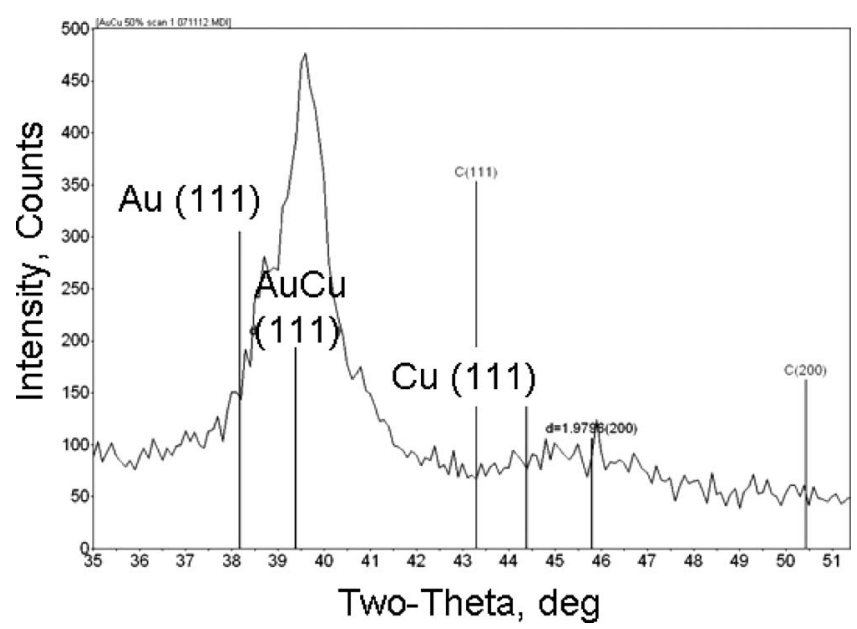

FIG. 1. XRD for $100 \mathrm{~nm}$ thick $\mathrm{Au}_{50} \mathrm{Cu}_{50}$ alloy.

namic conductivity with angle of incidence in the $\mathrm{Au}$ and $\mathrm{Cu}$ films.

The dc electronic transport in the films was characterized using four point probe measurements. Using the known relationship between dc conductivity and relaxation time as in Eq. (4), the dc relaxation time was measured using standard values for the constants $n$ and $m^{*}$. A valence of one free electron per atom was assumed to determine carrier concentration, and the effective mass was taken to be 0.99 and 1.49 times the standard electron mass for $\mathrm{Cu}$ and $\mathrm{Au}$, respectively. ${ }^{22}$ Measured relaxation times were then used in the Drude model for comparison with ellipsometer measurements. Experiments described in Sec. IV were conducted to alter the relaxation time and measure the resulting change in the dynamic conductivity. For each experiment both ellipsometric and dc electronic transport measurements were made.

\section{RESULTS}

\section{A. dc electronic transport four point probe measurements}

The first experiment was to decrease the relaxation time by increasing the impurity scattering density in a $\mathrm{Au}-\mathrm{Cu}$ alloy. Compositions of $\mathrm{Au}_{75} \mathrm{Cu}_{25}, \mathrm{Au}_{50} \mathrm{Cu}_{50}$, and $\mathrm{Au}_{25} \mathrm{Cu}_{75}$ in atomic percent were used to test a range of relaxation times. Boron was added to an additional alloy at a composition of $\mathrm{Au}_{50} \mathrm{Cu}_{40} \mathrm{~B}_{10}$ to further increase scattering. The films were deposited by cosputtering $\mathrm{Au}$ and $\mathrm{Cu}$ with deposition rates set relative to the desired composition in a UHV PVD tool. The thicknesses of the films were approximately $100 \mathrm{~nm}$. No thermal processing was performed on the films and the crystal structure was measured by x-ray diffraction (XRD) to be the disordered fcc phase in each case. The films may then be considered to be a random mixture of $\mathrm{Au}, \mathrm{Cu}$, or $\mathrm{B}$ atoms with no intermetallic phase present. XRD data demonstrating the fcc phase for the $\mathrm{Au}_{50} \mathrm{Cu}_{50}$ alloy are shown in Fig. 1 and the dc electronic transport measurements for the range of $\mathrm{Au}-\mathrm{Cu}$ films are in Table I. The increase in dc resistivity in the alloy corresponded to the relaxation time falling to just $14 \%$ of the pure $\mathrm{Au}$ relaxation time for the $\mathrm{Au}_{50} \mathrm{Cu}_{50}$ film, and further decreases to $5.7 \%$ for the $\mathrm{Au}_{50} \mathrm{Cu}_{40} \mathrm{~B}_{10}$ film. The (111) peak in the XRD data shifted to an intermediate value 
TABLE I. dc electronic transport measurements including maximum and minimum $\omega \tau$ products for IR band.

\begin{tabular}{|c|c|c|c|c|}
\hline Metal & $\begin{array}{c}\rho_{\mathrm{dc}} \\
(\mu \Omega \mathrm{cm})\end{array}$ & $\begin{array}{c}\tau \\
(\mathrm{fs})\end{array}$ & $\omega \tau, \tau=20 \mu \mathrm{m}$ & $\omega \tau, \tau=2 \mu \mathrm{m}$ \\
\hline $\mathrm{Au}, 295 \mathrm{~K}$ & 4.21 & 13.8 & 1.30 & 13.0 \\
\hline $\mathrm{Cu}, 295 \mathrm{~K}$ & 4.48 & 9.33 & 0.879 & 8.79 \\
\hline $\mathrm{Cu}$ annealed at $295 \mathrm{~K}$ & 1.97 & 25.0 & 2.36 & 23.6 \\
\hline $\mathrm{Au}, \sim 4 \mathrm{~K}$ & 1.21 & 50.0 & 4.71 & 47.1 \\
\hline $\mathrm{Cu}$ annealed at $\sim 4 \mathrm{~K}$ & 0.198 & 304 & 28.6 & 286 \\
\hline $\mathrm{Au}_{50} \mathrm{Cu}_{50}, 295 \mathrm{~K}$ & 23.3 & 2.12 & 0.200 & 2.00 \\
\hline $\mathrm{Au}_{75} \mathrm{Cu}_{25}, 295 \mathrm{~K}$ & 10.2 & 5.31 & 0.500 & 5.00 \\
\hline $\mathrm{Au}_{25} \mathrm{Cu}_{75}, 295 \mathrm{~K}$ & 12.6 & 4.93 & 0.464 & 4.64 \\
\hline $\mathrm{Au}_{50} \mathrm{Cu}_{40} \mathrm{~B}_{10}, 295 \mathrm{~K}$ & 71.7 & 0.862 & 0.0812 & 0.812 \\
\hline
\end{tabular}

between that of the pure components, and the broad Lorentzian character of the peak indicated a high defect density consistent with the decreased relaxation time.

The second experiment aimed to increase relaxation time by measuring dynamic conductivity at cryogenic temperature. The cryogenic dc electronic transport measurements were carried out by immersing the samples in liquid helium and then measuring with an in situ four point probe. Both a $\mathrm{Au}$ and $\mathrm{Cu}$ samples were measured and the results are given in Table I where relaxation time has been calculated based on the dc resistivity. The increase in relaxation time is a factor of 6 larger for the $\mathrm{Cu}$ film compared to the $\mathrm{Au}$ film because the $\mathrm{Cu}$ film was annealed at $400 \mathrm{C}$ for $30 \mathrm{~min}$. The heat treatment decreased the defect density in the $\mathrm{Cu}$ film thereby reducing the residual resistivity when the phonon contribution to resistivity was removed at low temperature. The resistivity decrease is comparable to those previously observed in similar films. ${ }^{23}$

\section{B. Ellipsometric dynamic conductivity measurements}

Contrary to dc conductivity results, the increase in impurity scattering in the $\mathrm{Au}_{50} \mathrm{Cu}_{50}$ film was found to result in larger dynamic conductivity compared to the pure components as shown in Fig. 2. When the product of the frequency and relaxation time $\omega \tau$ for $\mathrm{Au}_{50} \mathrm{Cu}_{50}$ was equal to unity at a wavelength of $4 \mu \mathrm{m}$, the $\mathrm{Au}_{50} \mathrm{Cu}_{50}$ alloy's dynamic conduc-

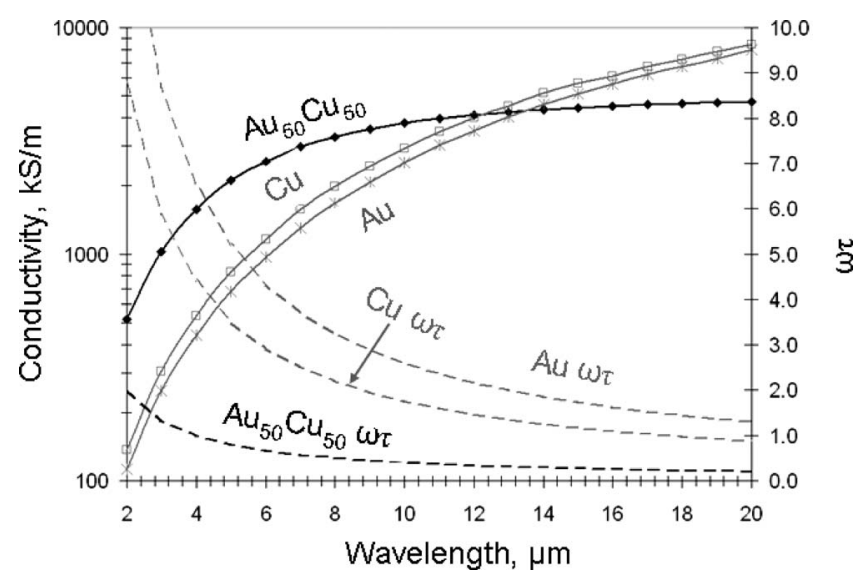

FIG. 2. Ellipsometric dynamic conductivity data for $\mathrm{Au}_{50} \mathrm{Cu}_{50}$ alloy compared to pure components shown as solid lines and the $\omega \tau$ products for each shown as broken lines.

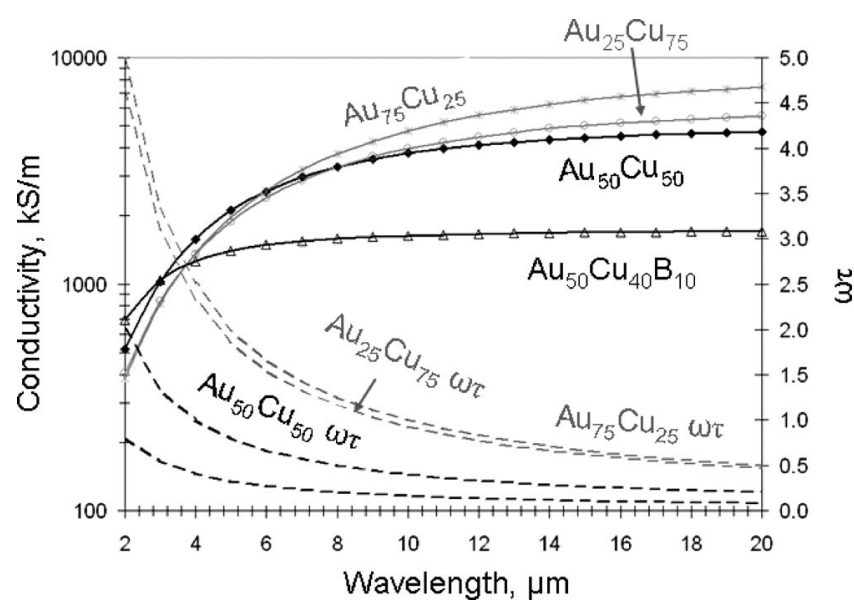

FIG. 3. Ellipsometric dynamic conductivity data for range of alloy films including $\mathrm{Au}_{75} \mathrm{Cu}_{25}, \mathrm{Au}_{50} \mathrm{Cu}_{50}, \mathrm{Au}_{25} \mathrm{Cu}_{75}$, and $\mathrm{Au}_{50} \mathrm{Cu}_{40} \mathrm{~B}$ 10 as solid lines with $\omega \tau$ products for each as broken lines.

tivity was greater than that of $\mathrm{Cu}$ by $195 \%$ and of $\mathrm{Au}$ by $260 \%$. At shorter wavelengths the dynamic conductivity of the alloy continued to increase relative to the pure components. At sufficiently long wavelengths, the pure components had a higher conductivity than $\mathrm{Au}_{50} \mathrm{Cu}_{50}$ once the $\omega \tau$ product was far enough below unity. This occurs when the product is equal to 0.33 . This counterintuitive result of increased conductivity with decreased electronic mean free path is in qualitative agreement with the Drude model.

The measured dynamic conductivities of the full range of alloys are shown in Fig. 3. There existed a range of wavelengths for which each type of alloy had the highest dynamic conductivity relative to the other alloys. At wavelengths longer than $7 \mu \mathrm{m}$ the $\mathrm{Au}_{75} \mathrm{Cu}_{25}$ or $\mathrm{Au}_{25} \mathrm{Cu}_{75}$ alloys had the largest dynamic conductivity corresponding to their smaller dc resistivities. At wavelengths ranging from 3 to $7 \mu \mathrm{m}$, the $\mathrm{Au}_{50} \mathrm{Cu}_{50}$ alloy was found to have the largest dynamic conductivity. The $\mathrm{Au}_{50} \mathrm{Cu}_{40} \mathrm{~B}_{10}$ alloy was found to have the greatest dynamic conductivity at wavelengths shorter than $3 \mu \mathrm{m}$. In each case the $\omega \tau$ product ranges from about 0.4 to 1.4 in the range of greatest dynamic conductivity. This suggests that an ideal relaxation time exists for any given wavelength to maximize the dynamic conductivity. An expression for the ideal relaxation time is derived in Sec. V and tested against the alloy dynamic conductivity data.

The absence of phonon scattering at cryogenic temperature decreased the dynamic conductivity in the $\mathrm{Au}$ and $\mathrm{Cu}$ films as shown in Fig. 4. Due to the lack of thermal processing in the Au film the increase in $\omega \tau$ is smaller than that of the $\mathrm{Cu}$ film; however, the decrease in dynamic conductivity is greater for the $\mathrm{Au}$ than for the $\mathrm{Cu}$ film. Over the 2 to $20 \mu \mathrm{m}$ band the $\omega \tau$ product decreased monotonically from 13.5 to 1.35 at $395 \mathrm{~K}$ and from 47.1 to 4.71 at $4 \mathrm{~K}$ as shown in Table I. Similarly the $\omega \tau$ product decreased monotonically in the $\mathrm{Cu}$ film from 28.8 to 2.88 at $295 \mathrm{~K}$ and from 287 to 28.7 at $4 \mathrm{~K}$. At approximately $4 \mathrm{~K}$ the dynamic conductivity of Au decreased to $22 \%, 31 \%$, and $47 \%$ of its value at $295 \mathrm{~K}$ at wavelengths of 4,10 , and $20 \mu \mathrm{m}$, respectively. The dynamic conductivity of $\mathrm{Cu}$ decreased to $51 \%, 48 \%$, and $56 \%$ of its $295 \mathrm{~K}$ value at wavelengths of 4,10 , and $20 \mu \mathrm{m}$, 


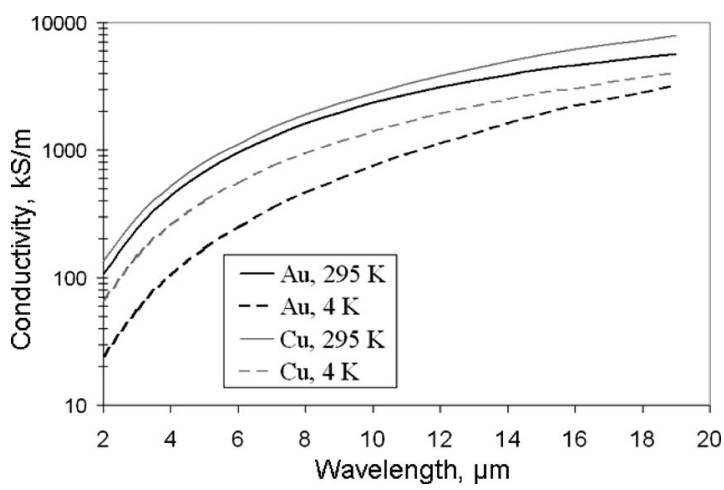

FIG. 4. Ellipsometric dynamic conductivity data under cryogenic temperatures. Solid lines refer to data at $295 \mathrm{~K}$ and broken lines refer to data at approximately $4 \mathrm{~K}$.

respectively. The cryogenic data showed that the IR dynamic conductivity had the opposite response of the dc conductivity to increases in electronic mean free path. The experiments with impurity scattering also showed the same opposing response.

\section{DISCUSSION}

The measured increases in dynamic conductivity were observed when the $\omega \tau$ product was increased to a value of around 0.4 or higher. Although these results are in qualitative agreement with the Drude model, there are quantitative differences between the measurements and the classical theory. This is represented in Fig. 5 with the Drude deviation function $\gamma(\omega)$ defined as the ratio between the measured dynamic conductivity data and the Drude modeled conductivity defined in Eq. (4). The relaxation times measured using the four point probe and the constants discussed in the experiment section were used when calculating Eq. (4). The results may be interpreted using the anomalous skin effect and dynamic relaxation time theories as defined in Eq. (7). This resulted in a fitting function with two independent constants shown in Eq. (8). Expressions for $\alpha_{1}$ and $\alpha_{2}$ are given in Eq. (9).

$$
\begin{aligned}
& \gamma(\omega)=\frac{\sigma(\omega)_{\text {measured }}}{\sigma(\omega)_{\text {Drude }}}=\frac{\alpha_{1}}{\sqrt[3]{\omega}}+\alpha_{2} \omega^{5 / 3}, \\
& \alpha_{1}(\beta)=\frac{1}{\tau_{\mathrm{dc}}}\left(\frac{\beta}{V_{F}}\right)^{2 / 33} \sqrt{\frac{2 m^{*}}{n e^{2} \mu_{0}}}
\end{aligned}
$$

\begin{tabular}{|c|c|c|c|c|c|c|c|c|}
\hline Metal & $\beta$ & $\begin{array}{c}b \\
\text { (fs) }\end{array}$ & Mean error $(\%)$ & Standard deviation (\%) & $\begin{array}{c}\lambda, 3 \mu \mathrm{m} \\
\left(n_{\mathrm{eff}} / n\right)\end{array}$ & $\begin{array}{c}\lambda, 10.6 \mu \mathrm{m} \\
\quad\left(n_{\mathrm{eff}} / n\right)\end{array}$ & $\begin{array}{l}\lambda, 3 \mu \mathrm{m} \\
{[\tau(\mathrm{fs})]}\end{array}$ & $\begin{array}{l}\lambda, 10.6 \mu \mathrm{m} \\
{[\tau(\mathrm{fs})]}\end{array}$ \\
\hline $\mathrm{Au}, 295 \mathrm{~K}$ & 0.766 & 0.0577 & 9.63 & 5.72 & 0.558 & 0.849 & 10.5 & 13.5 \\
\hline $\mathrm{Cu}, 295 \mathrm{~K}$ & 0.447 & 0.0720 & 3.83 & 2.03 & 0.619 & 0.941 & 7.37 & 9.14 \\
\hline $\mathrm{Cu}$ annealed at $295 \mathrm{~K}$ & 9.07 & 0.0336 & 12.90 & 12.00 & 1.50 & 2.29 & 18.8 & 24.4 \\
\hline $\mathrm{Au}, \sim 4 \mathrm{~K}$ & 5.31 & 0.00653 & 3.76 & 1.95 & 0.154 & 0.235 & 44.0 & 49.5 \\
\hline $\mathrm{Cu}$ annealed at $\sim 4 \mathrm{~K}$ & 5840 & 0.00116 & 2.71 & 1.74 & 0.0189 & 0.0289 & 267 & 301 \\
\hline $\mathrm{Au}_{50} \mathrm{Cu}_{50}, 295 \mathrm{~K}$ & 0.0819 & 0.0278 & 2.33 & 2.74 & 0.815 & $\sim 1$ & 2.07 & 2.12 \\
\hline $\mathrm{Au}_{75} \mathrm{Cu}_{25}, 295 \mathrm{~K}$ & 0.207 & 0.255 & 5.50 & 5.20 & 0.608 & 0.926 & 3.46 & 5.09 \\
\hline $\mathrm{Au}_{25} \mathrm{Cu}_{75}, 295 \mathrm{~K}$ & 0.191 & 0.378 & 6.95 & 5.55 & 0.581 & 0.885 & 2.84 & 4.65 \\
\hline $\mathrm{Au}_{50} \mathrm{Cu}_{40} \mathrm{~B}_{10}, 295 \mathrm{~K}$ & 0.0142 & 0.385 & 5.00 & 6.95 & 0.622 & 0.947 & 0.762 & 0.853 \\
\hline
\end{tabular}

TABLE II. Fitted values and associated error relative to wavelength where indicated.

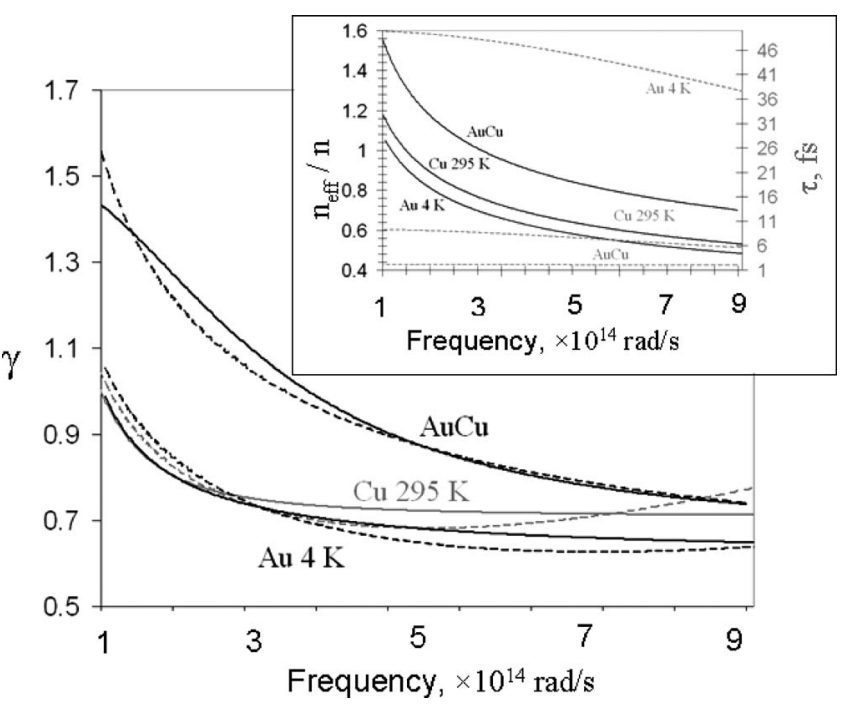

FIG. 5. Drude deviation ratios for selected metals; solid lines refer to measured data and broken lines refer to fitted data. Inset: solid lines refer to $\frac{n_{\text {eff }}}{n}$ and broken lines refer to relaxation time.

$$
\alpha_{2}(\beta, b)=b\left(\frac{\beta}{V_{F}}\right)^{2 / 33} \sqrt{\frac{2 m^{*}}{n e^{2} \mu_{0}}} .
$$

The fitted functions are compared to the measured data in Fig. 5 for selected data, and results from the full range of experiments are shown in Table II. The discrepancy between the fitted and measured functions was computed as a function of frequency and this was taken to be the error in the fitting process. The mean and standard deviations of the error data set were calculated and are shown in Table II. Using known values for the constants in Eqs. (7) and (9), the dynamic relaxation time and effective carrier concentrations were determined as shown in Table II and plotted for selected data in Fig. 5. The unknown values in Eq. (7) include and the constant $\beta$ from the theory of the anomalous skin effect. The fitted values for both constants are shown in Table II. The constant $\beta$ was directly proportional to the electronic mean free path as expected from its definition in Ref. 16. The quadratic term $b$ was small compared to the dc relaxation time as expected, and it was also inversely proportional to the electronic mean free path. The frequency dependent behavior of all the experiments were consistent with that shown in Fig. 5, so no further information is required to the quadratic term from the dynamic relaxation time theory 
describe the behavior of the films beyond that shown in Table II. Instances where the effective carrier concentration increased above unity were considered to be a result of the model becoming invalid when the anomalous skin effect did not occur. This behavior is seen in the long-wavelength spectra of the size effect and $\mathrm{AuCu}$ films, which had little anomalous skin effect to begin with due to their small electronic mean free paths.

The mean error and standard deviations were consistently low across the experiments with the exception of the room temperature $\mathrm{Au}$ films, which tended to be somewhat higher. The values in Table II show that adding scattering via impurities in $\mathrm{Au}-\mathrm{Cu}$ alloys increased the effective carrier concentration, and that removing phonon scattering via testing at cryogenic temperatures significantly decreased the effective carrier concentration. The effective carrier concentration is lower for the $\sim 4 \mathrm{~K} \mathrm{Cu}$ film compared to the $\sim 4 \mathrm{~K}$ $\mathrm{Au}$ film due to the larger grain size of the $\mathrm{Cu}$ film. At $295 \mathrm{~K}$ data for both annealed and unprocessed $\mathrm{Cu}$ are shown in Table II. As expected the annealed $\mathrm{Cu}$ film has a longer electronic mean free path, which results in a larger value for $\beta$ compared to the unprocessed $\mathrm{Cu}$ film.

The changes in the quadratic term $b$ in Table II suggest a connection between electronic mean free path and dynamic relaxation time. In general as impurities in the metal film increase, electrons scatter more as a function of frequency. This implies that the mechanism behind dynamic relaxation time is neither electron-electron scattering nor a two-carrier mechanism, but that electrons scatter off of the same obstacles at high frequency as they do in de transport. However, at high frequency there is a greater relaxation time penalty when the impurity or defect density increases. The electron-electron scattering mechanism should be independent of crystal structure or impurities, but this was clearly not the case from the data in Table II. Although the annealed $\mathrm{Cu}$ film may be assumed to have larger grains than the unprocessed films, the unannealed films should have similar grain sizes near that of their common thicknesses. Thus a two-carrier model involving grain boundaries cannot explain the dynamic relaxation time data in Table II. We conclude that electronic scattering in the IR is analogous to scattering in dc transport, but in the IR scattering occurs over a shorter time scale.

The results for impurity scattering in alloy films showed that there was a range of frequencies over which each film had a dc relaxation time that produced the greatest dynamic conductivity. These results suggest that there exists an ideal dc relaxation time that yields the largest possible dynamic conductivity at a particular frequency for a given metal. By using the Drude model in Eq. (4) it can be shown that the greatest dynamic conductivity occurs when the dc relaxation time is equal to the inverse of the frequency. This would imply that the ideal relaxation time is independent of the relationship between the electronic mean free path and skin depth of the metal. A correction factor from the theory of the anomalous skin effect should then be added to the Drude model prediction of the ideal relaxation time. This yields the

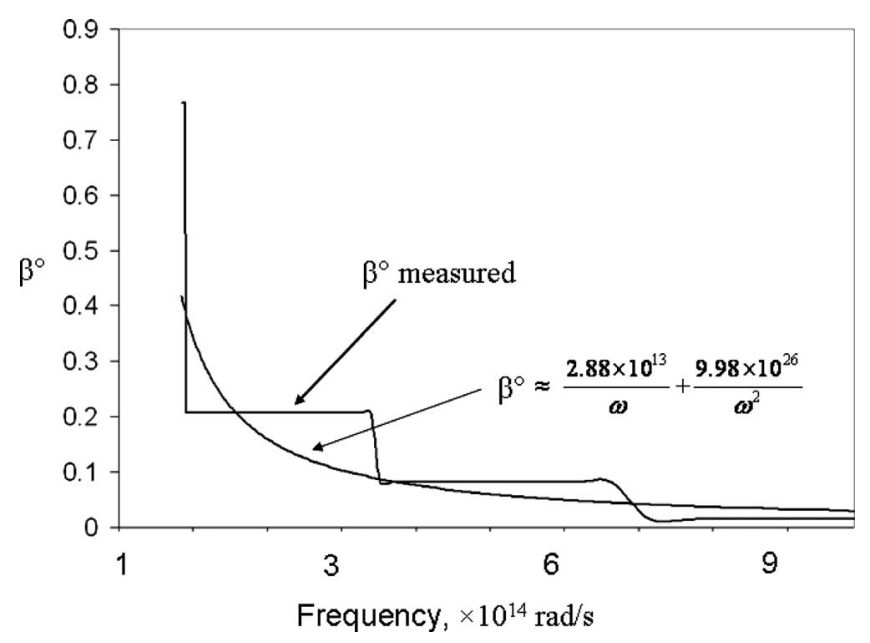

FIG. 6. The reference $\beta^{\circ}(\omega)$ that is taken to be the measured $\beta$ constant corresponding to the alloy with the highest dynamic conductivity over a given frequency range and fitted to a Taylor series in Eq. (13).

expression in Eq. (10) where the ratio of effective to dc carrier concentration is used as the anomalous skin effect correction factor.

$$
\tau_{\text {ideal }}^{3}=\left(\frac{n_{\text {eff }}}{n} \frac{1}{\omega}\right)^{3}=\frac{\beta}{V_{F}} \sqrt{\left(\frac{2 m^{*}}{n e^{2} \mu_{0}}\right)} \omega^{-2} .
$$

In Eq. (10) $\mathrm{n}_{\text {eff }}$ has been substituted from Eq. (5). In order to establish convenient design rules for IR metamaterials and antennas, Eq. (10) may be rewritten to give the ideal dc resistivity as a function of frequency.

$$
\rho_{\text {ideal }}^{3}=\left(\frac{V_{F}}{\beta}\right) \omega^{2} \sqrt{\frac{\mu_{0}}{2}}\left(\frac{m^{*}}{n e^{2}}\right)^{5 / 2} \text {. }
$$

Although $\beta$ is not a function of frequency, $\beta$ varies as the electronic mean free path is adjusted by the addition of impurities. In order to quantitatively determine the ideal relaxation time for a particular frequency, the value of $\beta$ that corresponds to the alloy with the greatest dynamic conductivity must be used. This reference $\beta$ will be called $\beta^{\circ}(\omega)$ for clarity. Thus an empirical $\beta^{\circ}(\omega)$ plot may be constructed from the measured data by taking the $\beta$ values that corresponded to the alloy with greatest dynamic conductivity for a given wavelength range. Using the available data points for the $\mathrm{Au}-\mathrm{Cu}$ system a step function is generated for $\beta^{\circ}(\omega)$ as shown in Fig. 6. To aid calculation, the experimental data can be fitted by considering that $\beta$ is proportional to the relaxation time, which tends to go toward $\omega^{-1}$ at the ideal relaxation time. Following this reasoning the data were fit to a Taylor series in Eq. (12), and this is shown in Fig. 6.

$$
\beta^{\circ}(\omega) \cong n_{0}+\frac{n_{1}}{\omega}+\frac{n_{2}}{\omega^{2}}+\cdots
$$

In Eq. (13) a simplified expression for the ideal dc resistivity $\rho_{\text {ideal }}$ as a function of wavelength in $\mu \mathrm{m}$ is obtained in units of $\mu \Omega \mathrm{cm}$ using the fitted $\beta^{\circ}$. 


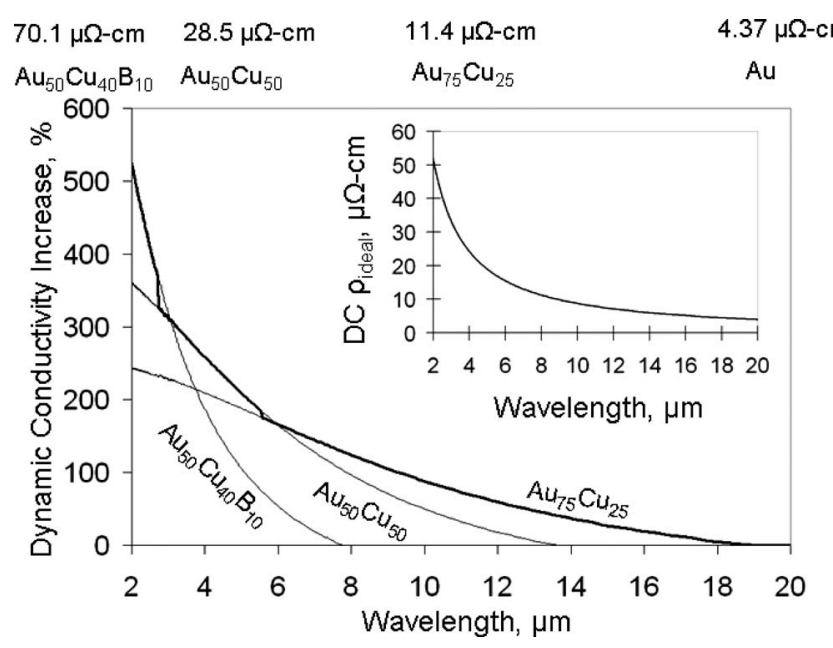

FIG. 7. Dynamic conductivity increase is plotted for each alloy (in at. \%) compared to pure Au. Bold line highlights alloy with the greatest conductivity increase based ideal resistivity in Eq. (13) compared to measured values shown at the top of the figure.

$$
\begin{gathered}
\rho_{\text {ideal }}(\mu \Omega-\mathrm{cm})=\frac{34.1}{0.248 \lambda(\mu \mathrm{m})+0.0654 \lambda(\mu \mathrm{m})^{4 / 3}} \\
\quad \text { for } \mathrm{Au}-\mathrm{Cu} \text { system. }
\end{gathered}
$$

The alloy film experiment may then be used to verify the prediction of Eq. (11). Figure 7 shows the percent increase in dynamic conductivity as a function of wavelength for $\mathrm{Au}$ with different concentrations of $\mathrm{Cu}$ or $\mathrm{B}$ impurities. The bolded black line in Fig. 7 highlights the dynamic conductivity increase in the film that was predicted by Eq. (11) to have the greatest dynamic conductivity over the wavelength band. The alloy composition and resulting dc resistivity are displayed for each band at the top of Fig. 7. The ideal dc resistivity based on Eq. (13) is plotted in the inset to Fig. 7.

There is good agreement between the ideal relaxation time predicted at a given frequency by Eq. (13) and the measured relaxation time for the alloy with the greatest conductivity at the corresponding frequency. This may be seen by comparing the ideal relaxation time in the inset to Fig. 7 to the measured values shown at the top of Fig. 7 for a given wavelength range. Because of the dependence in Eq. (11) on the surface scattering term $\beta$, the ideal resistivity is selfcorrecting of changes in temperature and impurity or defect densities. Thus Eq. (11) will have useful applications in designing metal films for antennas and metamaterials with the greatest possible dynamic conductivity at any wavelength or operating temperature.

\section{CONCLUSION}

Decreasing the relaxation time was proven to be an effective method for increasing the dynamic conductivity of noble metals in the IR band. These effects resulted from an increase in the effective carrier concentration. The increase in dynamic conductivity was strongest at shorter wavelengths below $8 \mu \mathrm{m}$ where the anomalous skin effect was more pronounced. The dynamic conductivity in the 3-5 $\mu \mathrm{m}$ wavelength bands was shown to increase by more than
$300 \%$, and below $3 \mu \mathrm{m}$ the dynamic conductivity increased by more than $500 \%$. There was also shown to be a corresponding decrease in dynamic conductivity at cryogenic temperatures to only $20 \%$ of its room temperature value in the $3-5 \mu \mathrm{m}$ wavelength bands. The results of these experiments may be applied to improving the performance of IR antenna systems. A relationship was established for the ideal $\mathrm{dc}$ relaxation time or resistivity to produce the greatest possible dynamic conductivity as a function of both frequency and the fraction of effective electrons from the anomalous skin effect. By adjusting for the anomalous skin effect the ideal dc resistivity is able to take the effects of both temperature and impurity scattering into account. These theories have been verified by experiments and may be used to customize metallic alloy films for antenna elements and metamaterials with the optimal performance at the design wavelength. Antenna systems in the 3-5 $\mu \mathrm{m}$ bands or shorter wavelengths would particularly benefit from these enhanced materials as would IR systems operating in cryogenic environments such as outer space.

\section{ACKNOWLEDGMENTS}

The authors would like to thank J. Menendez, V. R. D'Costa, and C. Poweleit at the Arizona State University Department of Physics for their assistance with the cryogenic IR ellipsometry measurements.

${ }^{1}$ B. Monacelli, J. Pryor, B. A. Munk, D. Kotter, and G. D. Boreman, IEEE Antennas Wireless Propag. Lett. 53, 745 (2005).

${ }^{2}$ D. J. Shelton, J. S. Tharp, G. Zummo, W. R. Folks, and G. D. Boreman, J. Vac. Sci. Technol. B 25, 1827 (2007).

${ }^{3}$ J. C. Ginn, B. Lail, D. J. Shelton, J. S. Tharp, W. R. Folks, and G. D. Boreman, Appl. Comput. Electromagn. Soc. J. 22, 184 (2007).

${ }^{4}$ D. A. Bonn, J. E. Greedan, C.V. Stager, T. Timusk, M. G. Doss, S. L. Herr, K. Kamarás, and D. B. Tanner, Phys. Rev. Lett. 58, 2249 (1987).

${ }^{5}$ C. M. Chorey, K. S. Kong, K. B. Bhasin, J. D. Warner, and T. Itoh, IEEE MTT-S Int. Microwave Symp. Dig. 39, 1480 (1991).

${ }^{6}$ L. Novotny, Phys. Rev. Lett. 98, 266802 (2007).

${ }^{7}$ J. B. Pendry, L. Martin-Moreno, and F. J. Garcia-Vidal, Science 305, 847 (2004).

${ }^{8}$ H. Raether, Surface Plasmons on Smooth and Rough Surfaces (Springer, Heidelberg, 1988).

${ }^{9}$ G. Fahsold, A. Bartel, O. Krauth, N. Magg, and A. Pucci, Phys. Rev. B 61, 14108 (2000).

${ }^{10}$ G. Fahsold, M. Sinther, A. Priebe, S. Diez, and A. Pucci, Phys. Rev. B 70, 115406 (2004).

${ }^{11}$ M. Fox, Optical Properties of Solids (Oxford University, New York, 2000), 143-160.

${ }^{12}$ R. Lenk and A. Knabchen, J. Phys.: Condens. Matter 5, 6563 (1993).

${ }^{13}$ J. M. Ziman, Principles of the Theory of Solids, 2nd ed. (Cambridge University Press, New York, 1972), p. 282.

${ }^{14}$ A. B. Pippard, Proc. R. Soc. London, Ser. A 224, 273 (1954).

${ }^{15}$ E. H. Sondheimer, Adv. Phys. 50, 499 (2001).

${ }^{16}$ R. G. Chambers, Proc. R. Soc. London, Ser. A 215, 481 (1952).

${ }^{17}$ H. E. Bennett, J. M. Bennett, E. J. Ashley, and R. J. Motyka, Phys. Rev. 165, 755 (1968).

${ }^{18}$ S. R. Nagel and S. E. Schnatterly, Phys. Rev. B 9, 1299 (1974).

${ }^{19}$ G. R. Parkins, W. E. Lawrence, and R. W. Christy, Phys. Rev. B 23, 6408 (1981).

${ }^{20}$ F. Forstmann and R. R. Gerhardts, Metal Optics Near the Plasma Frequency (Springer, Berlin, 1986), p. 46.

${ }^{21}$ P. Zhou, S. Y. Wang, J. Li, R. J. Zhang, H. Y. You, Z. C. Shen, and L. Y. Chen, Thin Solid Films 455-456, 157 (2004).

${ }^{22}$ P. B. Johnson and R. W. Christy, Phys. Rev. B 6, 4370 (1972).

${ }^{23}$ T. Sun, B. Yao, A. P. Warren, V. Kumar, S. Roberts, K. Barmak, and K. R. Coffey, J. Vac. Sci. Technol. A 26, 605 (2008). 